\title{
Mathematical Modeling of the Spread of HIVIAIDS by Markov Chain Process
}

\author{
Rotich Kiplimo Titus \\ Department of Center for Teacher Education, Moi University, Eldoret, Kenya
}

Email address:

tisesko@yahoo.com

\section{To cite this article:}

Rotich Kiplimo Titus. Mathematical Modeling of the Spread of HIV/AIDS by Markov Chain Process. American Journal of Applied Mathematics. Vol. 4, No. 5, 2016, pp. 235-246. doi: 10.11648/j.ajam.20160405.15

Received: September 6, 2016; Accepted: September 23, 2016; Published: October 15, 2016

\begin{abstract}
The spread of the Human Immunodeficiency Virus (HIV) and the resulting Acquired Immune Deficiency syndrome (AIDS) is a major health concern. Mathematical models are therefore commonly applied to understand the spread of the HIV epidemic. In this study, HIV dynamics is analyzed using a Stochastic Discrete-Time Markov Chain Mathematical Model. Demographic and epidemiological parameters that affect the model population dynamics were investigated. Well posedness of the model determined and the conditions for the existence and stability of disease-free and endemic equilibrium points proved, using the next generation matrix technique. The effect of various intervention strategies, were simulated by varying the parameters representing the possible strategies and comparing the respective values of the reproductive ratio $R_{0}$. The numerical simulation results using intervention transition matrix showed that vertical transmission is the most sensitive parameter standing at 0.6 followed by the use of HAART at 0.4 . This indicates the strategy which requires much effort to avert progression of infected individual to AIDS.
\end{abstract}

Keywords: Markov Chain, Reproductive Ratio, Stability, Transition Matrix

\section{Introduction}

Acquired Immune Deficiency Syndrome (AIDS) is an infectious disease caused by Human Immunodeficiency Virus (HIV) retrovirus. The retrovirus targets and infects a type of immune cells containing $\mathrm{CD} 4^{+}$proteins on its surface, hence called $C D 4^{+} T$ cells or briefly called T cells. Once HIV has infected these T cells, the HIV genome is integrated with $\mathrm{T}$ cell DNA and during cell division, many other HIV is produced, infecting many other cells. On the other hand, the immune system will destroy infected cells and gradually, the $\mathrm{T}$ cells decline to levels unable to protect the body against opportunistic diseases such as pneumonia, meningitis, cancers and tuberculosis. It is then that an infected individual is said to have developed AIDS [15].

The HIV epidemic has been a major cause of morbidity and mortality worldwide. The rate of the spread of the HIV/AIDS epidemic has reached a shocking level. On a global scale, the HIV epidemic has stabilized, although with unacceptably high levels of new infections and AIDS deaths. Based on the current trends, over 7300 persons become infected with HIV, and 5400 die from AIDS-related causes including more than 760 children, every day. This means five people are becoming infected with HIV every minute and four people dying from AIDS per minute (UNAIDS, 2009). AIDS is the leading cause of death in Sub-Saharan Africa, especially in the southern part of the continent where nine countries with the highest HIV prevalence worldwide are all located in this sub region, with each of these countries experiencing adult HIV prevalence greater than $10 \%$. With an estimated adult HIV prevalence of $26 \%$ in 2007 , Swaziland has the most severe level of infection in the world [26]. The recent statistics have shown that an estimate of 22.4 million [20.8 million - 24.1 million] people (women account for approximately $60 \%$ ) were living with HIV in Sub-Saharan Africa at the end of 2008 . Moreover, $72 \%$ of world's AIDSrelated deaths, $68 \%$ of new HIV infections among adults and 91\% of new HIV infections among children occurred in SubSaharan Africa. In addition, the epidemic has left behind more than 14 million AIDS orphans in the region in 2008 [26].

It has been established that HIV is transmitted through three major ways, namely; sexual intercourse, blood transfusion and from mother-to-child. HIV transmission as a 
result of sexual intercourse accounts for about three-quarters of all HIV infection world-wide, with heterosexual transmission being the major means [9]. Proportion of HIV transmission through blood, for example as a result of the medical transfusion of infected blood or blood products or from the use of contaminated injection equipment by intravenous drug users and sharing of cutting tools and the vertical transmission from mother- to- child during pregnancy, delivery or through breast-feeding is also significant.

Since the emergence of HIV/AIDS in the 1980s, there is no drug or vaccine that can be used to control HIV/AIDS and the only available control strategy is behavioural and AntiRetro-viral (ARV) therapy. Behavioural methods include; adopting low risk behaviours, one sexual partner, faithfulness, use of condom, avoiding sharing of injections and cutting tools, regular testing and avoiding drug use. Treatment regimens or options are available for HIV that reduces viral loads and slow the progression to AIDS but none of these eradicate the virus or prevent the spread of infections. Use of ARVs by already infected individuals lessens the severity of infection by improving CD4+ T cell count and fighting opportunistic diseases. However, it does not treat HIV completely, and the virus will continue remaining in the system, with the victim remaining infective, thus creating a pool of potential transmitters of the disease. Lack of curative drugs leaves behavioral adjustment to prevent infection as the only option to curb the pandemic. Abstinence, Being faithful and Condom use (ABC) has been practiced earlier without success. The reason is attributed to insufficient public awareness campaign on effective use of available ARVs and $\mathrm{ABC}$, and unclear distribution of HIV/AIDS among the population. The awareness of how far the pandemic has spread helps both the public health and the public to improve on their safety precaution against contracting the disease.

Unlike the early years of AIDS epidemic where the majority of infected individuals were prostitutes, homosexuals, hemophiliacs, and intravenous drug users, today there is no geographical area, class and cultural group of the world untouched by this pandemic, with the epicenter of HIV pandemic in Sub-Saharan Africa. The in this region have the highest HIV prevalence worldwide, and experience adult HIV prevalence rates greater than 10\% [21]. For example, by 2008 , Swaziland had the most severe level of infection in the world with an estimated adult HIV prevalence of $26 \%$ in 2007 , [21].

Since the spread of HIV depends on the behavioural characteristics of the people, efforts by the public health to enlighten masses on the control of the spread of HIV through behaviour change have shown to significantly help control the dispersal gradient of the epidemic [4]. Because of this reason, the use of mathematical epidemiological models to understand the disease dispersal dynamics helps in analyzing the trends and prediction of future states and allocation of control strategy resources to curb the pandemic.

The use of mathematics to model communicable diseases dates back to the $18^{\text {th }}$ century. The first known result in mathematical epidemiology was on a defense of the practice of inoculation against smallpox in 1760 by Daniel Bernoulli. Other contributions to modern mathematical epidemiology are due to P.D. En'ko between 1873 and 1894 [14]. Mathematical modeling now plays a key role in policy making, including health-economic aspects; emergency planning and risk assessment; control-programme evaluation; and monitoring of surveillance data. In research, it is essential in study design analysis (including parameter estimation) and interpretation [13]. Many mathematical models including deterministic and stochastic models have used to describe the dynamics of infectious diseases. The most suitable model which takes into account environmental and demographic stochasticity is the stochastic model. Stochastic models assigns a probabilistic value to the parameters hence gives the exact picture of the situation. In this paper, the spread of HIV/AIDS is studied using Markov Chain process, incorporating stochasticity to determine the threshold values of the parameters that determine the gradient of HIV spread, in order to curb the negative effects of the pandemic. This model will suggest appropriate intervention strategies that guide the allocation of resources in finding a lasting solution to HIV/AIDS pandemic.

\section{Literature Review}

This section reviews literature on the transmission of HIV/AIDS and the use of mathematical models in describing infection dynamics.

\subsection{HIV Infection Stages}

When an individual is infected with HIV, the clinical response is complex, progressive and varies among individuals. Within few days of infection, an individual develops an acute mononucleosis like syndrome with fever, malaria and lymphadenopathy or the swelling of the lymph glands. These symptoms abate as HIV bonds to cells with $\mathrm{CD} 4+$ receptor protein on the surface. These CD4+T Lymphocyte cell levels will then drop rapidly from a preinfection normal level of about 1200 cells per $\mathrm{ml}$ to about 800 cells per ml [5]. Within 2 - 4 weeks after HIV infection, many, but not all, people develop flu-like symptoms often described as "the worst flu ever". Symptoms can include fever, swollen glands, sore throat, rash, muscle and joint aches, fatigue and headache. This primary infection is body's initial response to the HIV infection. This is called acute infection stage. During this early period of this stage, large amounts of virus are being produced by the body and consequently, CD4 cells are destroyed and their count fall rapidly. Eventually the immune response will begin to bring the level of virus in the body back down to a level called viral set point, which is relatively stable level of virus in the body. The next stage is the asymptomatic stage; people who are infected with HIV experience no HIV-related symptoms or mild ones. The HIV virus continues to reproduce at very low levels, although it is still active. The last is the AIDS 
stage. The immune system is badly damaged and become vulnerable to infections and infection-related cancers called opportunistic infection. In average, the number of $\mathrm{CD} 4^{+}$cells falls below 200 cells $/ \mathrm{mm}^{3}$ of blood. Right from the second stage, an infective is able to transmit the disease and infect others.

\subsection{Mathematical Modeling and Epidemiology}

Mathematical modeling has become an integral part of the research on the control of HIV infection, since it is capable to provide useful predictions that can be used as the basis for assessing the effects of chemotherapy or immunotherapy and for the design of optimum therapies and effective vaccines [1]. A mathematical model is a representation of actual phenomenon using mathematical concepts and language, analysis and interpretation of mathematical results to understand the real situation. This models can be applied to epidemiology, which is defined by [12] as the study of the distribution and determinants of diseases both infectious and non-infectious. Mathematical models have been used extensively in research into the epidemiology of HIV/AIDS, to help improve our understanding of major contributing factors to its spread [17]. The goal of any modeling exercise is to extract as much information as possible from available data and provide an accurate representation of both the known and uncertainties of the epidemic [19]. Mathematical models based on the underlying transmission mechanism of HIV help the medical and scientific community understand better how the disease spreads in the community and how changes in various parameter values affect the course of the epidemic. Therefore, by developing such mathematical models, the potential effectiveness of different approaches for bringing the epidemic under control, can be evaluated. Control of the epidemic therefore depends on the knowledge of such parameters which can be analyzed and their sensitivities simulated through mathematical epidemiological modeling. The effectiveness of the two HIV/AIDS control strategies; behavioral and therapeutic depends on the quantitative understanding of the dynamics of the epidemic in the population.

Many mathematical models are deterministic and subdivide the individuals into classes depending on their infection status. [23] studied the dynamics of HIV using deterministic SEIR model and obtained the basic reproductive number $\mathrm{R}_{0}$ which helped them to determine the global stability of disease free and endemic equilibrium. According to [8], the threshold for many epidemiological models is the basic reproduction number $\mathrm{R}_{0}$. [16] developed an age-structured model incorporating vertical transmission and showed that the only possible way to ensure a diseasefree equilibrium was to bring the force of infection to zero. They showed that it is possible to have a disease-free children group if all the babies born by infected mothers were HIV-free. However, this did not guarantee safety in the adult group, and this would only happen if the rate of vertical infection was zero. The alternative strategy of controlling HIV pandemic is the use of ARV therapy. A person under
ARV therapy uses drugs which interrupt the entry of HIV into the $\mathrm{T}$ cells and also interfere with replication. These ARVs are classified as Protease Inhibitors and Reverse Transcriptase Inhibitors respectively. Treatment with antiretroviral increases the life expectancy of people infected with HIV, with average survival time estimated to be more than 5 years as of 2005 [22]. Without antiretroviral therapy, someone who has HIV will develop AIDS and typically die within a year. The development of Highly Active AntiRetroviral Therapy (HAART) for HIV infection has substantially reduced the death rate of HIV victims [20]. As the life expectancy of persons with HIV has increased in countries where HAART is widely used, the continuing spread of the disease has caused the number of persons living with HIV to increase substantially. This means that an equilibrium point need to be stricken, to balance between increasing potential pool of transmitters and reducing new cases of infections. [17] developed a model of transmission of HIV into population of varying size and other demographical and epidemiological factors. In their analysis, they found that an increase in the rate of transmission leads to increase the population of infective which in turn increases the AIDS population. Thus, they concluded that the spread of the disease should be controlled by the way of promoting effective treatment to keep the overall infective population under control. This study utilized a Markov chain model to predict the trends of the epidemic.

Forecasting the progression of HIV/AIDS spread plays an important role in controlling disease transmission and alleviating health disparities. Given the growing threat of limited resources in the society, especially in the health care system and public health system, the projection of the future epidemic can help to optimize resource allocation and design efficient, economical, timely health policies targeting the high risk populations and high prevalence areas.

According to [10] a Markov process is a type of stochastic processes in which a system changes in a random manner between different states, at regular or irregular intervals. Markov chain modeling has been applied to a number of studies in the medical field over the years to predict and estimate random or uncertain events associated with specific probabilities of occurrence. Some practical applications have been observed in analysis of genetics, particularly in sickle cell anaemia, determining efficacy of noninsulin-dependent diabetes in a population of patients, predicting outcomes of dialysis treatment/kidney transplants in patients and analyzing longitudinal disease progression for liver cancer breast cancer, bronchiolitis obliterans syndrome and Alzheimer's disease, among others. [6] used Markov chain model to track the movement of the HIV dynamics virus from one generation to another in a period of 20 years. They applied multistate Markov modeling to explain the rate at which the Hepatitis C disease progresses. Observational data on 1306 patients in Trent, England was collected from 1991 to 2006 and used for the longitudinal study. They also developed a multivariate Markov chain model to project tuberculosis (TB) progression in the US from the 1980s to 
2010 among different races in the country.

Although previous studies have modeled disease progress and transmission dynamics using Markov models, few have focused on epidemiological disease progression at a macro level of total populations through Markov models. The prediction of both incidence and prevalence of HIV/AIDS, made using the Markov model, will help in planning and calibrating adequate surveillance systems, as well as guiding public health in selecting and mobilizing suitable intervention and treatment strategic plans.

A three-state Markov model was used to described survival functions from smoking cessation interventions [25]. It was found that on quitting, smokers transit through a state of withdrawal characterized by a high rate of relapse, and then into a more secured state of long - term abstinence. The Markov model embodies the dynamic nature of the cessation/relapse process; it permits stronger inference to long - term abstinence rates, provides measures of treatment efficacy, describes the outcomes of new quit attempts, and suggests mechanisms for the survival process.

Markov micro simulation model accurately assess the cost-

$$
\begin{gathered}
S(t+1)=\Lambda+(1-(\alpha+\gamma)) r S(t)-\mu_{1} S(t)+\operatorname{mrI}(t) \\
I(t+1)=q(\alpha+\gamma) r S(t)-\mu_{2} I(t)+(1-\rho)(1-m) r I(t)+\theta r A(t) \\
A(t+1)=(1-q)(\alpha+\gamma) r S(t)+\rho(1-m) r I(t)-\mu_{3} A(t)+(1-\theta) r A(t)
\end{gathered}
$$

where the transfer parameters $\Lambda \geq 0, \alpha \geq 0, \gamma \geq$ $0, \rho \geq 0, \mu_{1} \geq 0, \mu_{2} \geq 0, \mu_{3} \geq 0, m \geq 0, \theta \geq 0, r \geq 0 \quad$ and $q \geq 0$. The first equation represents population of susceptibles who are recruited at a constant rate $\Lambda$, and augmented by successfully treated infectives at a rate $m$. These individuals are transferred to other compartments through vertical infection at birth $\alpha$ and horizontal infection due to sexual intercourse at a rate $\gamma=\beta \frac{I}{N}$ and lost by natural death rate of $\mu_{1}$. The second difference equation accounts for the infective population, with a proportion $q(\alpha+\gamma)$ recruited from the susceptible class after getting infection and additional from AIDS class who recover at a rate $\theta$ due to effective use of ARVs and treatment of opportunistic diseases. Individuals in this class are lost because of accelerated death at a rate $\mu_{2}$ and transferred to AIDS class at a rate of $\rho(1-m)$ due to unsuccessful treatment of infectives. The third equation models AIDS class who receive individuals directly from infected susceptibles at a rate $(1-q)$ and from infective class with probability of

$$
\begin{gathered}
S\left(t_{1}\right)=\Lambda+(1-(\alpha+\gamma)) r S_{0}-\mu_{1} S_{0}+m r I_{0} \\
I\left(t_{1}\right)=q(\alpha+\gamma) r S_{0}-\mu_{2} I_{0}+(1-\rho)(1-m) r I_{0}+\theta r A_{0} \\
A\left(t_{1}\right)=(1-q)(\alpha+\gamma) r S_{0}+\rho(1-m) r I_{0}-\mu_{3} A_{0}+(1-\theta) r A_{0}
\end{gathered}
$$

With $\quad 0 \leq \alpha \leq 1,0 \leq r \leq 1,0 \leq \mu_{1} \leq 1,0 \leq \rho \leq 1,0 \leq$ $m \leq 1,0 \leq \theta \leq 1,0 \leq \mu_{3} \leq 1$, and that $0 \leq I(t) \leq N(t)$ for all $t \geq 0, t \in \mathbb{N}$, then $0 \leq[1-(\alpha+\gamma)] \leq 1$ or $0 \leq$ $(\alpha+\gamma) \leq 1$. Let $\mu=\max \left\{\mu_{1}, \mu_{2}, \mu_{3}\right\}$, and let $p=(r-\mu)>$ 0 be the survival rate. Then the total population $N\left(t_{1}\right)=$ $S\left(t_{1}\right)+I\left(t_{1}\right)+A\left(t_{1}\right)$, satisfies the relation, $\rho(1-m)$ of those not successfully treated.

\subsection{Positivity and Boundedness}

Due to biological considerations, we require that all solutions of system (1) are positive. Define a bounded invariant space, $\mathbb{R}_{+}=\{S, I, A \mid S>0, I>0, A>0\} \quad$ and $\mathbb{R}_{+0}=\left\{S(0)=S_{0} \geq 0, I(0)=I_{0} \geq 0, A(0)=A_{0} \geq 0, S+\right.$ $\left.I+A=N \leq \frac{\Lambda}{1-\left(r-\mu_{1}\right)}\right\}$.

Proposition 1. (Positivity of Solutions) The solutions $S(t), I(t), A(t)$ of system (1) with initial values from $\mathbb{R}_{+0}$ are positive for all $t \geq 0$. The space $\mathbb{R}$ is positively invariant and all solutions starting in $\mathbb{R}$ approach enter or remain in $\mathbb{R}$.

Proof: Let the initial conditions be $S(0)=S\left(t_{0}\right)=S_{0} \geq$ $0, I(0)=I\left(t_{0}\right)=I_{o} \geq 0, A(0)=A\left(t_{0}\right)=A_{0} \geq 0 \in \mathbb{R}_{+0}$, then using system (1), we have the solutions at the next time interval $t_{1}=t_{0}+h$ ( $h$ as the step size) as,

$$
N\left(t_{1}\right)=\Lambda+p N\left(t_{0}\right)
$$

whose solution is given for all $t \geq 0$ by

$$
N(t)=\frac{\Lambda\left(1-p^{t}\right)}{1-p} N(0)+p^{t} N(0)
$$


Clearly, the limit of $N(t)$ in equation (4) as $t \rightarrow \infty$ tends to a positive fixed point defined as

$$
N^{*}=\frac{\Lambda}{1-\left(\mathrm{r}-\mu_{1}\right)}
$$

This implies that the solutions are positive and bounded for all positive time $0 \leq t \leq \infty$, that is, all solutions starting in the region $\mathbb{R}$ approach, enter or stay in $\mathbb{R}$.
There are usually at least two fixed points in epidemiology, namely; Disease Free Equilibrium (DFE) and Endemic Equilibrium Point (EEP). The DFE occurs in absence of the disease, while EEP occurs when the disease persist.

\subsubsection{Disease Free Equilibrium (DFE)}

Define system (1) in vector form as, $X_{t+1}=A X$ where, $X=(S, I, A)^{T}$ and the matrix $A$ is a $3 \times 3$ matrix given by;

\subsection{Fixed Points}

$$
A=\left(\begin{array}{ccc}
(1-(\alpha+\gamma)) r-\mu_{1} & m r & 0 \\
q(\alpha+\gamma) r & (1-\rho)(1-m) r-\mu_{2} & \theta r \\
(1-q)(\alpha+\gamma) r & \rho(1-m) r & (1-\theta) r-\mu_{3}
\end{array}\right)
$$

The DFE $\bar{X}$ of systems (1) is obtained by solving $\bar{X}=A \bar{X}$ with $I^{0}=0, A^{0}=0$ is given by

$$
\bar{X}:=\left(S^{0}, I^{0}, A^{0}\right)=\left(\frac{\Lambda}{1-\left(r-\mu_{1}\right)}, 0,0\right)
$$

\subsubsection{Endemic Equilibrium Point (EEP)}

Solving $\bar{X}=A \bar{X}$ with $I^{0} \neq 0, A^{0} \neq 0$ using equation (1) yields the EEP of system (1) defined by $\left(S^{e}, I^{e}, A^{e}\right)$ as;

$$
\left(\frac{\Lambda}{\left(1-r+\mu_{1}+r K\right)-m r},-\frac{\left(1-r+\mu_{1}+r K\right)}{(m r)^{2}}, \frac{\Lambda(1-q) r K}{\left[\left(1-r+\mu_{1}+r K\right)-m r\right]\left(\mu_{3}+\theta\right)}-\frac{\rho(1-m) r\left(1-r+\mu_{1}+r K\right)}{\left(\mu_{3}+\theta\right)(m r)^{2}}\right)
$$

\subsection{Extinction and Persistence of the Disease}

Extinction or persistence of the disease depends on the reproductive ratio $R_{0}$. Considering only the disease compartments, that is; a compartments with infected individuals, we eliminate $S(t)$ in system (1) using the relation $S(t)=N^{*}-I(t)-A(t)$. This yields a reduced system of two equations,

$$
\begin{gathered}
I_{(t+1)}=q \beta \frac{I_{(t)}}{N^{*}} r\left(N^{*}-I_{(t)}-A_{(t)}\right)-\mu_{2} I_{(t)}+(1-\rho)(1-m) r I_{(t)}+\theta r A_{(t)} \\
A_{(t+1)}=(1-q) \beta \frac{I_{(t)}}{N^{*}} r\left(N^{*}-I_{(t)}-A_{(t)}\right)+\rho(1-m) r I_{(t)}-\mu_{3} A_{(t)}+(1-\theta) r A_{(t)}
\end{gathered}
$$

with positive invariant manifold defined as; $\Omega=\left\{(I, A) \in R_{+}^{2} \mid I \geq 0, A \geq 0, I+A<\frac{\Lambda}{1-\left(r-\mu_{1}\right)}\right\}$.

\subsubsection{Reproductive Ratio}

The definition and computation of the basic reproductive number $R_{0}$ is done using the next generation matrix approach described by [7], [24]. Using this approach, the basic reproductive ratio for system (8) is found to be;

$$
R_{0}=\frac{q r \beta\left[1+\mu_{3}-(1-\theta) r\right]+(1-q) r \beta \theta r}{\left[1+\mu_{2}-(1-\rho)(1-m) r\right]\left[1+\mu_{3}-(1-\theta) r\right]+\theta r \rho(1-m) r}
$$

\subsubsection{Local Stability and $R_{0}$.}

Proposition 2. (Local Stability about the fixed points). With $R_{0}$ as a measure of replacement rate of the infection, if $R_{0}<1$, the DFE of model (1) is the only equilibrium point and is Locally Asymptotically Stable (LAS) with the disease extinction at the limit as $t \rightarrow \infty$. If $R_{0}>1$, DFE is unstable and there exist another fixed point, EEP where the disease persists in the population.

Proof: The stability matrix $M$ extracted by linearizing system (1) is given by

$$
M=\left(\begin{array}{ccc}
\left(1-\beta \frac{I}{N}\right) r-\mu_{1} & \left(1-\beta \frac{1}{N}\right) r S+m r & 0 \\
q \beta \frac{I}{N} r & q \beta \frac{S}{N} r-\mu_{2}+(1-\rho)(1-m) r & \theta r \\
(1-q) \beta \frac{I}{N} r & (1-q) \beta \frac{S}{N} r+\rho(1-m) r & -\mu_{3}+(1-\theta) r
\end{array}\right)
$$

Stability of system (1) is determined by the value of the eigenvalues of matrix $M$ evaluated at the equilibrium points. With the closed form solution of system (1) given by, $\bar{X}_{n}=D^{n} \bar{X}_{0}$, where $D=P^{-1} M P:=\operatorname{diag}(\lambda)$, we require that the magnitude of 
the eigenvalues $\lambda_{k}$ of matrix $M$ are less than one. The system is stable at DFE, $\left(S^{0}, I^{0}, A^{0}\right):=\left(\frac{\Lambda}{1-\left(r-\mu_{1}\right)}, 0,0\right)$ if $\left|\lambda_{k}\right|<1$. That is;

$$
\left|\begin{array}{ccc}
r-\mu_{1}-\xi & \frac{r \Lambda}{1-\left(\mathrm{r}-\mu_{1}\right)}+m r & 0 \\
0 & q \beta r+(1-\rho)(1-m) r-\mu_{2}-\xi & \theta r \\
0 & (1-q) \beta r+\rho(1-m) r & (1-\theta) r-\mu_{3}-\xi
\end{array}\right|=0
$$

Clearly, the first eigenvalue $\xi_{1}=\left|r-\mu_{1}\right|<1$. The other two eigenvalues are obtained from the quadratic characteristic equation

$$
\xi^{2}-\operatorname{Tr} a(M) \xi+\operatorname{Det}(M)=0
$$

Where $\operatorname{Tr} a(M)=$ Trace of $M=q \beta r+(1-\rho)(1-$ $m) r-\mu_{2}+(1-\theta) r-\mu_{3} \quad$ and $\quad \operatorname{Det}(M)=[q \beta r+$ $\left.(1-\rho)(1-m) r-\mu_{2}\right]\left[(1-\theta) r-\mu_{3}\right]-\theta r[(1-q) \beta r+$ $\rho(1-m) r]$ denotes the Determinant of matrix $M$.

The characteristic roots of Equation (10) are; $\xi_{1}=$ $\frac{1}{2} \operatorname{Tr} a(M)+\left[\frac{1}{4} \operatorname{Tr} a(M)^{2}-\operatorname{Det}(M)\right]^{\frac{1}{2}}$ and $\xi_{2}=\frac{1}{2} \operatorname{Tr} a(M)-$ $\left[\frac{1}{4} \operatorname{Tra}(M)^{2}-\operatorname{Det}(M)\right]^{\frac{1}{2}}$ with both $\left|\xi_{1}\right|<1$ and $\left|\xi_{2}\right|<1$. The conditions for stability of system (1) is to have the trace $\operatorname{Tr} a(M)>0$ and the determinant $\operatorname{Det}(M)>0$. This is satisfied if the condition $R_{0}+1<2 p$, therefore the system (10) is stable at EEP if;

$$
R_{0}<2 p-1
$$

\subsection{Sensitivity Analysis}

The threshold parameter $R_{0}$ which determines stability is a function of many parameters which include $m, \rho, \theta, q, \alpha$ which are the intervention parameters for treatment, use of ARVS, immune boosters, anti AIDS and PMCT respectively.

The sensitivity of these parameters is determined by computing the partial derivative of $R_{0}$ with respect to each parameter.

The sensitivity of $\alpha$ (Use of PMCT) is evaluated as;

$$
\frac{\partial R_{0}}{\partial \alpha}=\frac{q r\left[1+\mu_{3}-(1-\theta) r\right]+(1-q) r^{2} \theta}{\left[1+\mu_{2}-(1-\rho)(1-m) r\right]\left[1+\mu_{3}-(1-\theta) r\right]+\theta r \rho(1-m) r}:=s_{\alpha}
$$

The sensitivity of $\theta$ is evaluated as,

$$
\frac{\partial R_{0}}{\partial \theta}=\frac{\left\{q \beta r^{2}+(1-q) r^{2} \beta\right\} V-U\left\{\left[1+\mu_{2}-(1-\rho)(1-m) r\right] r+\rho(1-m) r^{2}\right\}}{V^{2}}:=s_{\theta}
$$

where $U=q r \beta\left[1+\mu_{3}-(1-\theta) r\right]+(1-q) r \beta \theta r$ and

$$
V=\left[1+\mu_{2}-(1-\rho)(1-m) r\right]\left[1+\mu_{3}-(1-\theta) r\right]+\theta r \rho(1-m) r
$$

The sensitivity of $m$ is evaluates as;

$$
\frac{\partial R_{0}}{\partial m}=-\frac{\left\{q \beta r\left[1+\mu_{3}-(1-\theta) r\right]+(1-q) r^{2} \beta \theta\right\}\{(1-\rho) r\}\left[1+\mu_{3}-(1-\theta) r\right] \theta r^{2} \rho}{\left\{\left[1+\mu_{2}-(1-\rho)(1-m) r\right]\left[1+\mu_{3}-(1-\theta) r\right]+\theta r \rho(1-m) r\right\}^{2}}:=s_{m}
$$

while the sensitivity of the $R_{0}$ with respect to $q$ will be;

$$
\frac{\partial R_{0}}{\partial q}=\frac{\beta r\left[1+\mu_{3}-(1-\theta) r\right]-r^{2} \beta \theta}{\left[1+\mu_{2}-(1-\rho)(1-m) r\right]\left[1+\mu_{3}-(1-\theta) r\right]+\theta r \rho(1-m) r}:=S_{q}
$$

and finally the sensitivity of $R_{0}$ with respect to $\rho$ is defined by;

$$
\frac{\partial R_{0}}{\partial \rho}=\frac{\{q r \beta \vartheta+(1-q) r \beta \theta r\}\{(1-m) r \vartheta+\theta r(1-m) r\}}{\left\{\left[1+\mu_{2}-(1-\rho)(1-m) r\right] \vartheta+\theta r \rho(1-m) r\right\}^{2}}:=s_{\rho}
$$

where $\vartheta=\left[1+\mu_{3}-(1-\theta) r\right]$. The sensitivity values of these parameters and their graphs are displayed in the next chapter.

\subsection{Simulation of Intervention Strategies}

In order to determine the effects of various intervention strategies, we simulate the results by varying the parameters representing the possible strategies. In the model system (2), the possible intervention strategies include;

\subsubsection{Prevention of Vertical Transmission}

This involves prevention of vertical transmission of HIV from mother to child through PMCT campaigns. It is represented by the rate parameter $0 \leq \alpha \leq 1$. When $\alpha=0$, all children are born free from HIV, while $\alpha>0$ means some children are infected either during birth or during breastfeeding by an infected mother. The available intervention strategy is more of behavioural than treatment. This is because all babies are born free from HIV and the mothers' behaviour may lead to the infection $f$ the child. Vaccines can also be used to prevent the child from getting infected.

\subsubsection{ARV Treatment of Infectives to Prevent Development of AIDS}

This is represented by the parameter $0 \leq q \leq 1$. This parameter varies with the use of ARV's. The greater the value of $q$, the more the infected people living with HIV and the lesser there are those with AIDS. This parameter is determined by the drug efficacy and accessibility to ARVs specifically the use of Zidovidine, Didanosine and others which inhibits the replication of virus, thus enabling the immune system to maintain sufficient CD4 cells. 


\subsubsection{Treatment of Infectives to Full Recovery}

This is quite minimal and currently very rare to find an individual who has successfully been cured and free from HIV. This is because the HIV virus resides in the cell nucleous and uses the cell mechanism to replicate. It can remain inside a dormant cell for life and thus it is not easy to cure an individual completely. However, if the HIV cells have been reduced to undetectable levels, then we can say the individual is cured. This treatment is denoted by the parameter $0 \leq m \leq 1$ and is facilitated by the use of a combined AntiRetroviral Therapy (cART).

\subsubsection{Progression to AIDS Class of Infected Patients}

AIDS cases can be treated by use of HAART, making the victims recover back into infective class. The probability of progression to AIDS class is represented by parameter $0 \leq \rho \leq 1$. A successfully treated individual $\rho=0$ recovers from AIDS class back to infective class (PLWH). The target of intervention is to reduce the value of $\rho$ to zero. The measure of the proportion of individuals who become successfully treated is denoted by $\theta$. The complementary proportion $(1-\theta)$ will remain with AIDS while the rest $\theta$ recover back to infectives.

\subsubsection{Force of Transmission}

The force of HIV transmission from one individual to the other is contributed generally by behavioral factors. These include high risk behaviours like multiple partners, unprotected sex, unfaithfulness, use of drugs just to mention but a few. All these determine the size of the parameter $0 \leq \beta \leq 1$. Any intervention strategy applied will have a reduction effect on the value of $\beta$. The smaller the value of $\beta$, the better the control of the spread of HIV pandemic. In this research, all the behavioural intervention strategies will be treated indiscriminately as one.

\subsection{Closed form Simulation System}

In order to determine the effects of change in parameters discussed above, the closed form solution of system (1) is used to determine the values of the next time interval by iteration. The model is given in matrix form as,

$$
\left(\begin{array}{c}
S(t+1) \\
I(t+1) \\
A(t+1)
\end{array}\right)=\left(\begin{array}{ccc}
(1-(\alpha+\gamma)) r-\mu_{1} & m r & 0 \\
q(\alpha+\gamma) r & (1-\rho)(1-m) r-\mu_{2} & \theta r \\
(1-q)(\alpha+\gamma) r & \rho(1-m) r & (1-\theta) r-\mu_{3}
\end{array}\right)\left(\begin{array}{c}
S(t) \\
I(t) \\
A(t)
\end{array}\right)
$$

or in compact form as

$$
\bar{X}_{t+1}=M \bar{X}_{t}
$$

where $M$ is the transition matrix and $\bar{X}$ is the vector $(S, I, A)^{T}$. The closed form solution of the system (17) is obtained after iterations as,

$$
\bar{X}_{n}=M^{n} \bar{X}_{n}
$$

During the implementation of any intervention strategy, the transformation matrix $M$ will be modified to increase $S(t+1)$ and reduce $I(t+1)$ and $A(t+1)$. Denoting the new transition matrix by $M_{c}$, then the new system with one occurrence of intervention will be

$$
\bar{X}_{n}=M_{c} M^{n-1} \bar{X}_{0}
$$

where the modified matrix will have the modified elements denoted with a subscript $c$ as

$$
M_{c}=\left(\begin{array}{ccc}
\left(1-\left(\alpha_{c}+\gamma_{c}\right)\right) r-\mu_{1} & m_{c} r & 0 \\
q_{c}\left(\alpha_{c}+\gamma_{c}\right) r & \left(1-\rho_{c}\right)\left(1-m_{c}\right) r-\mu_{2} & \theta_{c} r \\
\left(1-q_{c}\right)\left(\alpha_{c}+\gamma_{c}\right) r & \rho\left(1-m_{c}\right) r & \left(1-\theta_{c}\right) r-\mu_{3}
\end{array}\right)
$$

Applying the intervention strategy $p$ times yields

$$
\bar{X}_{n}=M_{c}^{p} M^{(n-p)} \bar{X}_{0}
$$

\section{Numerical Results}

The optimum intervention strategy is to apply the intervention strategy and maintain the steady state. This will help eliminate the HIV pandemic forever. The desired steady state is obtained when the intervention is applied continually to obtain the steady state matrix $M_{s}$ defined by the limit

$$
M_{S}=\lim _{p \rightarrow n}\left(\lim _{n \rightarrow \infty} M_{c}^{p} M^{(n-p)}\right)=\lim _{p \rightarrow \infty} M_{c}^{p}
$$

The numerical values and their graphical illustrations are presented in the next chapter to justify the theoretical results in this chapter.

We illustrate the theoretical analytic results in Chapter three by numerical simulation using the values presented in Table 1 below. The values were obtained from literature of [3], which were used to simulate HIV-TB co-infection. The parameter values were determined basing on data collected in Sub-Saharan Africa and thus a reflection to our Kenyan demographic and epidemiological situation. It is however recommended that, data from an area or location of interest must be collected and simulated using the MATLAB code provided in the appendix, before the model results are applied. 
The table below shows the parameter values used in the simulation, together with the initial conditions of the variables $\left(S_{0}, I_{0}, A_{0}\right)=(100,1,1)$. All parameters lie in the

interval $I=[0,1]$.

Table 1. Parameter Values for Model Simulation.

\begin{tabular}{llll}
\hline No & Parameter Description & Symbol & Value \\
\hline 1 & Constant Recruitment rate into the population & $\Lambda$ & 15 \\
2 & Infection during birth and/or through breastfeeding rate & $\alpha$ & 0.03 \\
3 & Disease transmission probability through risk behaviour & $\beta$ & 0.3 \\
4 & Probability that an Infective recover successfully and become Susceptible & $m$ & 0.02 \\
5 & Probability of successful treatment of an AIDS case back to Infectious state & $\theta$ & 0.5 \\
6 & Natural death rate of Susceptibles & $\mu_{1}$ & 0.02 \\
7 & Accelerated death rate due to infection & $\mu_{2}$ & 0.032 \\
8 & Accelerated death rate due to development of AIDS & $\mu_{3}$ & 0.05 \\
9 & Rate of becoming infectious once infected. (1-q) develop AIDS directly & $q$ & 0.8 \\
10 & Survival rate of a Susceptible & $r$ & 0.95 \\
11 & Natural progression of Infectives to AIDS & $\rho$ & 0.96 \\
\hline
\end{tabular}

\subsection{Markov Chain Mathematical Model}

The Markov Chain Mathematical Model is formulated from equation (2) and expressed in matrix form as in equation (17). Using the parameter values in Table 1, we obtain the model

$$
\left(\begin{array}{c}
S(t+1) \\
I(t+1) \\
A(t+1)
\end{array}\right)=\left(\begin{array}{ccc}
0.8984 & 0.0019 & 0 \\
0.0253 & 0.1576 & 0.8075 \\
0.0063 & 0.7585 & 0.0925
\end{array}\right)\left(\begin{array}{c}
S(t) \\
I(t) \\
A(t)
\end{array}\right)
$$

Notice that the sum of elements in each row does not add up to 1 . This is a modified matrix, applicable for a dynamical system, whose entire population changes with time. For a conserved system, where the population remains constant, the sum of elements of each column equals to one. In our case, the difference is the value of survival rate for each class, that is $s=r-\mu_{i}, i=1,2,3$. Using the provided initial conditions, $\left(S_{0}, I_{0}, A_{0}\right)=(100,1,1)$, the population distribution for the first 50 iterations of the system (18) yields

Table 2. The first 50 Iterations of System (20).

\begin{tabular}{|c|c|c|c|c|c|c|c|c|c|}
\hline $\mathbf{t}$ & $S(t+1)$ & $I(t+1)$ & $A(t+1)$ & $N(t+1)$ & $t$ & $S(t+1)$ & $\mathbf{I}(\mathrm{t}+\mathbf{1})$ & $\mathbf{A}(\mathrm{t}+1)$ & $N(t+1)$ \\
\hline 1 & 98 & 1 & 1 & 100 & 26 & 89.8858 & 48.837 & 42.099 & 180.8219 \\
\hline 2 & 103.0417 & 3.4453 & 1.471 & 107.958 & 27 & 88.62 & 49.746 & 42.9494 & 181.3153 \\
\hline 3 & 107.5756 & 4.3387 & 3.4012 & 115.3155 & 28 & 87.458 & 50.5651 & 43.7148 & 181.7378 \\
\hline 4 & 110.8257 & 6.8126 & 4.451 & 122.0893 & 29 & 86.3965 & 51.2982 & 44.4033 & 182.098 \\
\hline 5 & 113.526 & 8.3118 & 6.4899 & 128.3278 & 30 & 85.4304 & 51.954 & 45.0191 & 182.4034 \\
\hline 6 & 115.2569 & 10.8212 & 7.9723 & 134.0504 & 31 & 84.5544 & 52.5375 & 45.5692 & 182.6612 \\
\hline 7 & 116.4647 & 12.7391 & 10.094 & 139.2978 & 32 & 83.7628 & 53.0562 & 46.0583 & 182.8774 \\
\hline 8 & 116.9383 & 15.2773 & 11.8756 & 144.0913 & 33 & 83.0494 & 53.5155 & 46.4927 & 183.0575 \\
\hline 9 & 116.965 & 17.4509 & 14.0494 & 148.4653 & 34 & 82.4082 & 53.9214 & 46.8769 & 183.2065 \\
\hline 10 & 116.4555 & 19.9796 & 16.0067 & 152.4418 & 35 & 81.8334 & 54.2791 & 47.2162 & 183.3287 \\
\hline 11 & 115.601 & 22.2671 & 18.1828 & 156.0509 & 36 & 81.319 & 54.5938 & 47.515 & 183.4279 \\
\hline 12 & 114.3796 & 24.7297 & 20.2054 & 159.3147 & 37 & 80.8598 & 54.8699 & 47.7776 & 183.5073 \\
\hline 13 & 112.9224 & 27.0118 & 22.3255 & 162.2597 & 38 & 80.4505 & 55.1117 & 48.0078 & 183.5701 \\
\hline 14 & 111.2408 & 29.3482 & 24.3187 & 164.9077 & 39 & 80.0862 & 55.323 & 48.2094 & 183.6186 \\
\hline 15 & 109.4264 & 31.5298 & 26.3261 & 167.2824 & 40 & 79.7626 & 55.5072 & 48.3853 & 183.6551 \\
\hline 16 & 107.503 & 33.6869 & 28.2146 & 169.4045 & 41 & 79.4755 & 55.6676 & 48.5386 & 183.6816 \\
\hline 17 & 105.5342 & 35.6991 & 30.0622 & 171.2955 & 42 & 79.221 & 55.8068 & 48.6719 & 183.6997 \\
\hline 18 & 103.5444 & 37.6383 & 31.7918 & 172.9745 & 43 & 78.9958 & 55.9275 & 48.7876 & 183.7109 \\
\hline 19 & 101.5761 & 39.4378 & 33.447 & 174.4609 & 44 & 78.7967 & 56.0318 & 48.8878 & 183.7164 \\
\hline 20 & 99.6488 & 41.1382 & 34.985 & 175.772 & 45 & 78.621 & 56.1218 & 48.9744 & 183.7172 \\
\hline 21 & 97.7889 & 42.7047 & 36.4311 & 176.9247 & 46 & 78.4659 & 56.1994 & 49.0491 & 183.7143 \\
\hline 22 & 96.0089 & 44.162 & 37.7637 & 177.9346 & 47 & 78.3292 & 56.2659 & 49.1133 & 183.7085 \\
\hline 23 & 94.3233 & 45.4941 & 38.9989 & 178.8163 & 48 & 78.2089 & 56.323 & 49.1684 & 183.7003 \\
\hline 24 & 92.7379 & 46.7178 & 40.1276 & 179.5833 & 49 & 78.1031 & 56.3717 & 49.2155 & 183.6903 \\
\hline 25 & 91.2585 & 47.8279 & 41.1616 & 180.248 & 50 & 78.0101 & 56.4131 & 49.2558 & 183.679 \\
\hline
\end{tabular}

The term $N(t+1)$ is the total population of $S(t+1)+$ $I(t+1)+A(t+1)$. The graph below represents the population dynamics simulated in Table 2 . 


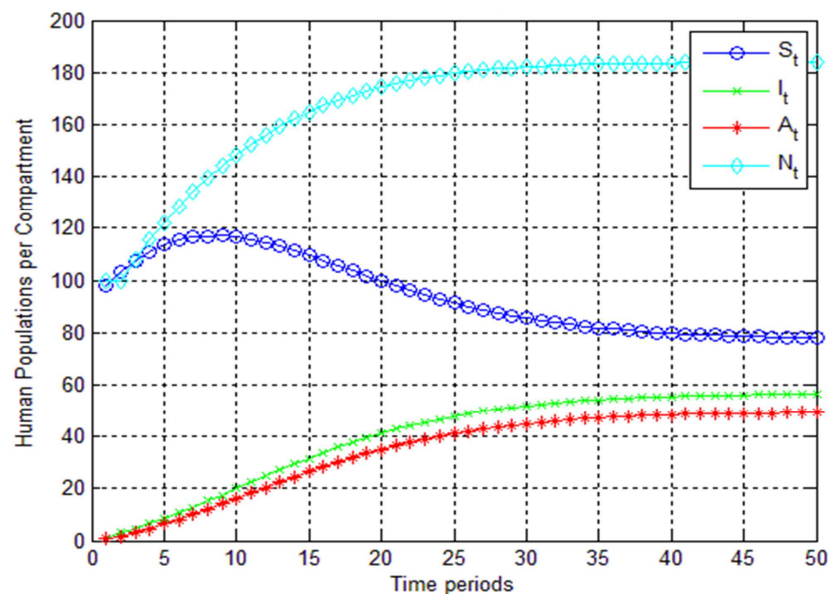

Figure 1. Cell Population Dynamics In absence of Intervention.

Clearly, the total population $N(t+1)$ continually increases as expected in normal life. Due to the introduction of one infective and one AIDS individual, the susceptibles continue to decrease especially after the infectives have increased significantly after 10 time intervals. The AIDS class follows soot increasing as Infectives increase. This is a plot of the values of $S(t+1), I(t+1), A(t+1)$ with the last values at time $t=50$ equal to $(S, I, A)=(78.0101,56.4131,49.2558)$ and $N=183.679$.

\subsection{Threshold Values of Intervention Parameters}

The parameters that determine the rate of transmission of the disease (HIV) as discussed in section 3.3 contribute to the value of $R_{0}$. The target of intervention strategy is to reduce $R_{0}$ to values less than one. This means that the infectives are not able to replace themselves and thus with time, the diseases will be phased out. The most important of the parameters is checked from the sensitivity analysis discussed in chapter three.

\subsection{Parameter Sensitivity Values}

Using the values of parameters presented in Table 1, the sensitivity values of intervention parameters are presented in Table 3 below.

Table 3. Table of Sensitivity Analysis Values.

\begin{tabular}{llll}
\hline No & Sensitivity Parameter Description & Symbol & Value \\
\hline 1 & $\begin{array}{l}\text { Effect of } \alpha \text { (rate of vertical infection) on the } \\
\text { value of } R_{0}\end{array}$ & $s_{\alpha}$ & 0.6123 \\
2 & $\begin{array}{l}\text { Effect of } \theta \text { (treatment of AIDS victim) on the } \\
\text { value of } R_{0}\end{array}$ & $s_{\theta}$ & 0.0069 \\
3 & $\begin{array}{l}\text { Effect of } m \text { (successful treatment of infective) } \\
\text { on the value of } R_{0}\end{array}$ & $s_{m}$ & 0.1830 \\
4 & $\begin{array}{l}\text { Effect of } q \text { (prevention of development of } \\
\text { AIDS) on the value of } R_{0}\end{array}$ & $s_{q}$ & 0.0444 \\
5 & $\begin{array}{l}\text { Effect of } \rho \text { (progression rate from } I_{t} \text { to } A_{t} \text { ) on } \\
\text { the value of } R_{0}\end{array}$ & $s_{\rho}$ & 0.4147 \\
\hline
\end{tabular}

The most sensitive parameter on the value of $R_{0}$ as shown in Table 3 above is $\alpha$ followed by $\rho$. This analysis indicates the target parameters for intervention. Clearly, since infants are helpless and depend entirely on their mothers to live, they are highly vulnerable and thus it is at this point that much care must be taken to prevent vertical transmission of HIV. Also, since there is no cure of HIV, the best strategy is to provide medication to prevent progression of infected individual to AIDS. These two intervention targets are the most important. The sensitivity graphs are illustrated in the figure below.

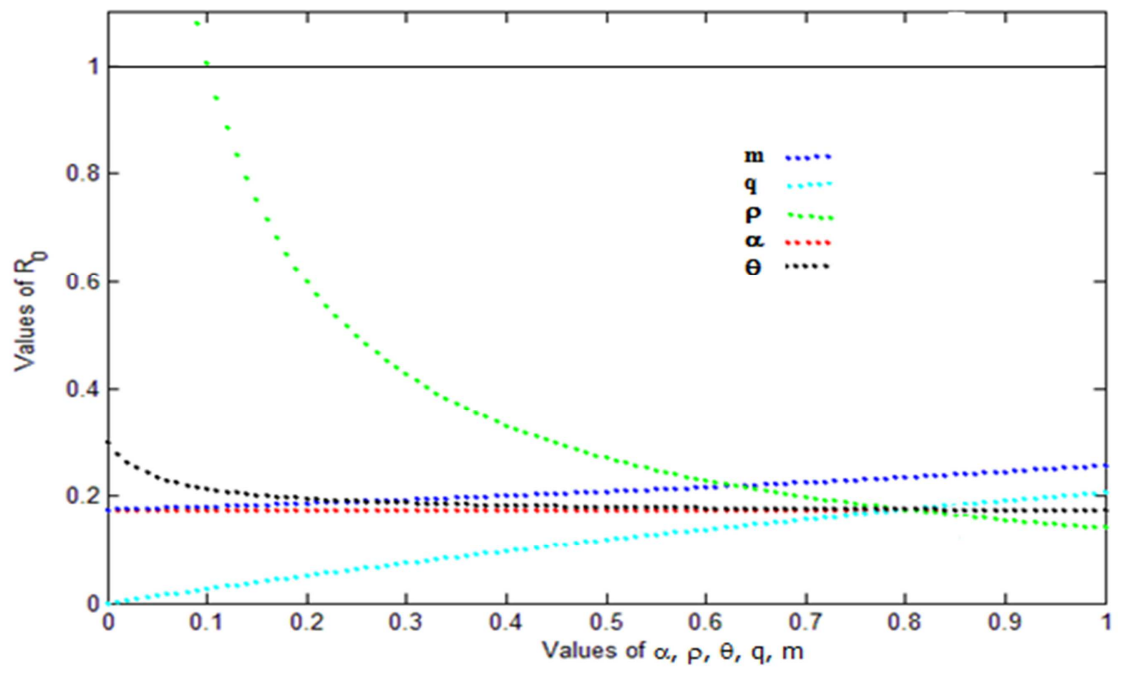

Figure 2. Sensitivity Analysis of intervention parameters.

\subsection{Intervention Matrix}

Due to intervention, the transition matrix $M_{c}$ in equation (19) will have the following elements

$$
M_{c}=\left(\begin{array}{ccc}
0.9063 & 0.0019 & 0 \\
0.0225 & 0.0015 & 0.9005 \\
0.0012 & 0.9596 & 0.0005
\end{array}\right)
$$

The interpretation of the intervention matrix is described as, $91 \%$ of susceptible will remain in susceptible class, while only $2.3 \%$ progress to infectives and none develop AIDS directly because of enhanced PMCT. The Infectives on the other hand will have none moving to susceptibles, because of absence of curative drugs, but with the use of ARVs, none will remain infective with $96 \%$ progressing to AIDS class. 
Lastly, the AIDS class will never move back to susceptibles, with majority $90 \%$ being treated back to susceptibles (PLWHA) and none remain with AIDS. This depicts an healthy nation with the steady state dynamics of the compartmental populations simulated in Table 3 below. This represents the solution of equation (19) of the form

$$
\lim _{n \rightarrow \infty} \bar{X}_{n}=M_{c}^{p} M^{(n-p)} \bar{X}_{0}
$$

Table 4. Simulation Results Using Intervention Transition Matrix.

\begin{tabular}{|c|c|c|c|c|c|c|c|c|c|}
\hline $\mathbf{t}$ & $S(t+1)$ & $I(t+1)$ & $\mathbf{A}(\mathrm{t}+\mathbf{1})$ & $N(t+1)$ & $\mathbf{T}$ & $S(t+1)$ & $I(t+1)$ & $A(t+1)$ & $N(t+1)$ \\
\hline 1 & 98 & 1 & 1 & 100 & 26 & 193.8856 & 0.719 & 0.6568 & 195.2614 \\
\hline 2 & 106.0023 & 1.0126 & 0.9431 & 107.958 & 27 & 195.215 & 0.6715 & 0.6783 & 196.5648 \\
\hline 3 & 113.433 & 0.9718 & 0.9557 & 115.3605 & 28 & 196.448 & 0.695 & 0.6338 & 197.7768 \\
\hline 4 & 120.3308 & 0.9961 & 0.918 & 122.2449 & 29 & 197.6017 & 0.6478 & 0.6556 & 198.9051 \\
\hline 5 & 126.7552 & 0.9527 & 0.9404 & 128.6483 & 30 & 198.6713 & 0.6717 & 0.6113 & 199.9543 \\
\hline 6 & 132.7282 & 0.9754 & 0.8997 & 134.6033 & 31 & 199.6728 & 0.6247 & 0.6336 & 200.9311 \\
\hline 7 & 138.2917 & 0.93 & 0.9207 & 140.1423 & 32 & 200.6008 & 0.649 & 0.5896 & 201.8394 \\
\hline 8 & 143.4636 & 0.9519 & 0.8781 & 145.2936 & 33 & 201.4707 & 0.6023 & 0.6122 & 202.6852 \\
\hline 9 & 148.2817 & 0.9052 & 0.8984 & 150.0853 & 34 & 202.2762 & 0.627 & 0.5685 & 203.4716 \\
\hline 10 & 152.7602 & 0.9267 & 0.8546 & 154.5415 & 35 & 203.032 & 0.5806 & 0.5914 & 204.204 \\
\hline 11 & 156.9331 & 0.8793 & 0.8745 & 158.6868 & 36 & 203.7314 & 0.6057 & 0.548 & 204.885 \\
\hline 12 & 160.8114 & 0.9006 & 0.83 & 162.542 & 37 & 204.3885 & 0.5596 & 0.5713 & 205.5194 \\
\hline 13 & 164.4259 & 0.8527 & 0.8498 & 166.1283 & 38 & 204.996 & 0.585 & 0.5282 & 206.1092 \\
\hline 14 & 167.7847 & 0.874 & 0.8048 & 169.4636 & 39 & 205.5676 & 0.5393 & 0.5518 & 206.6587 \\
\hline 15 & 170.916 & 0.8258 & 0.8247 & 172.5665 & 40 & 206.0955 & 0.5651 & 0.509 & 207.1695 \\
\hline 16 & 173.8253 & 0.8474 & 0.7795 & 175.4522 & 41 & 206.593 & 0.5196 & 0.533 & 207.6456 \\
\hline 17 & 176.5385 & 0.7991 & 0.7995 & 178.137 & 42 & 207.052 & 0.5458 & 0.4904 & 208.0882 \\
\hline 18 & 179.0588 & 0.8209 & 0.7542 & 180.6338 & 43 & 207.4854 & 0.5006 & 0.5147 & 208.5008 \\
\hline 19 & 181.41 & 0.7726 & 0.7744 & 182.957 & 44 & 207.8847 & 0.5271 & 0.4725 & 208.8843 \\
\hline 20 & 183.5936 & 0.7947 & 0.7292 & 185.1175 & 45 & 208.2625 & 0.4823 & 0.4971 & 209.2419 \\
\hline 21 & 185.6317 & 0.7465 & 0.7497 & 187.1279 & 46 & 208.61 & 0.5091 & 0.4552 & 209.5742 \\
\hline 22 & 187.524 & 0.7689 & 0.7046 & 188.9975 & 47 & 208.9396 & 0.4645 & 0.4801 & 209.8843 \\
\hline 23 & 189.291 & 0.7209 & 0.7254 & 190.7373 & 48 & 209.2423 & 0.4917 & 0.4384 & 210.1724 \\
\hline 24 & 190.9312 & 0.7437 & 0.6804 & 192.3553 & 49 & 209.5302 & 0.4474 & 0.4637 & 210.4413 \\
\hline 25 & 192.4636 & 0.6959 & 0.7015 & 193.8611 & 50 & 209.7940 & 0.4749 & 0.4223 & 210.6911 \\
\hline
\end{tabular}

The solution curves of the population dynamics under the intervention strategies will have the Susceptibles increasing while the infectives and AIDS case maintained at very low levels. The disease cannot be eradicated completely because the therapeutic drugs used are not to cure but to keep it very low. This scenario is illustrated in the figure below.

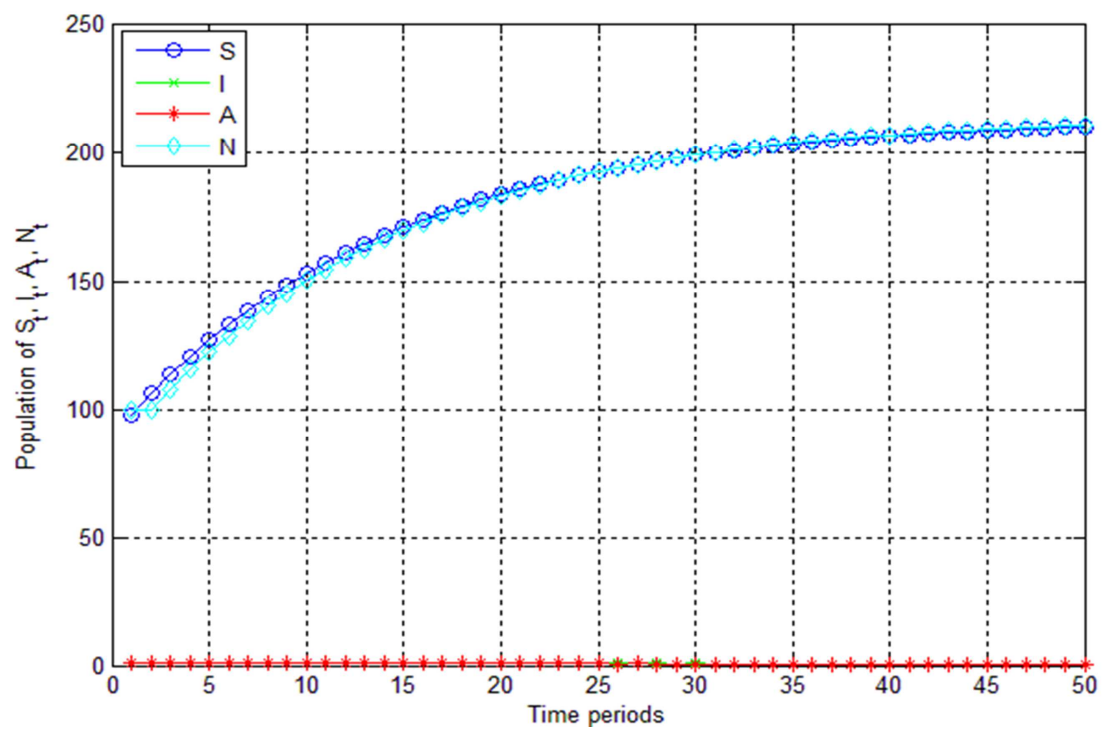

Figure 3. Population Dynamics with Intervention strategies.

From the Figure 3 above, it is clear that the only significantly large numbers of individuals are susceptibles. They are equal to the total population. The numbers of infectives and AIDS cases are very minimal.

\section{Conclusion}

In this study, a discrete-time Markov chain mathematical model has been proposed and analyzed to study the dynamic 
of HIV/AIDS model. The disease free and endemic equilibria are obtained and their stabilities investigated. A numerical study of the model has been conducted to see the impact of certain key parameters on the spread of HIV disease. It is observed that an increase in infectives through sexual intercourse, blood and from mother-to-child transmission would lead to increase the population of infectives which in turn increase the AIDS population.

\section{Intervention Strategies}

The following measures can be exercised in order to decrease the value of $R_{0}$ to be less than one so as to decrease the infections, which can lead to eradication or reduction of the HIV/AIDS disease;

\section{Use of Antiretroviral Drugs}

In many cases, an HIV-infected individual may be invaded by a host of common diseases ranging from very strong bacterial and viral infections to mild sicknesses. Since the infected person may be invaded by many infections simultaneously, it is noted that a single drug may not be sufficient to clear such an attack. As a result, a couple of drug recombinants that are particularly made to treat the HIV/AIDS patients of combinations of attacks have been developed. Individuals who have reached the advanced stages of HIV disease require combinations of antiretroviral drugs for their own health. This treatment, which must be taken every day for the rest of one's life, is also highly effective at preventing transmission of HIV.

\section{Prevention of Vertical transmission}

Effective prevention of mother-to-child transmission (PMTCT) is a threefold strategy (UNAIDS/WHO, 2005). Two strategies are under the discretion of the mother, while one is for the doctors to take initiative. The first is prevention of HIV infection among prospective parents. This involves taking HIV tests and other prevention interventions available in services related to sexual health such as antenatal and postpartum care. Providing appropriate counseling and support to women living with HIV will enable them to make informed decisions about their reproductive lives and help in preventing transmission of HIV from HIV-positive mothers to their unborn children during pregnancy, labour and at delivery. This calls for the integration of HIV care, treatment and support for women found to be positive and their families.

The second strategy is cesarean delivery. When a mother is HIV positive a cesarean section may be done to protect the baby from direct contact with her blood and other bodily fluids. There are indications that cesarean birth decreases the risk of mother-to-child transmission ([18]). If the mother is taking combination antiretroviral therapy then a cesarean section will often not be recommended because the risk of HIV transmission will be very low. But cesarean delivery may be recommended if the mother has a high levels of HIV concentration in her blood, although the procedure is seldom available and/or safe in resource poor settings ([2]).
The third PMTC strategy is safer infant feeding. A number of studies have shown that the protective benefit of drugs is diminished when babies continue to be exposed to HIV through breastfeeding [2]. Mothers with HIV are advised not to breastfeed but instead use breast milk substitutes (formula) which is acceptable, feasible, affordable, sustainable and safe. While weaning the baby, the newly introduced food corrodes the gut lining creating openings where HIV can penetrate. It is therefore advised that once the mother stops breastfeeding and introduce alternative baby food, HIV positive mothers should never breastfeed again because this will expose the child to high risk of infection.

\section{Recommendation}

Because of the continuity of HIV/AIDS epidemic worldwide, it is therefore important that the following recommendations should be taken on board by policy makers: more studies on assessment of treatment are required to provide more insight of dynamics of the disease to policy makers, intensification preventive strategies that will reduce risks with the infected HIV population and finally collection of appropriate data for modeling, accurate predictions and efficient planning.

\section{References}

[1] Adler, F. R., (1992) The effects of averaging on the basic reproduction ratio, Mathematical biosciences, 111, pp. 89-98.

[2] Brauer, F., (1995) Models for diseases with vertical transmission and nonlinear population dynamics, Mathematical biosciences, 128, pp. 13-24.

[3] Cao, H. and Tan, H., (2015) The discrete tuberculosis transmission model with treatment of latently infected individuals, Advances in Difference Equations, 2015, pp. 1.

[4] Cooley, P., Hamill, D., Liner, E., Myers, L. and Van Der Horst, C., (1993) A linked risk group model for investigating the spread of HIV, Mathematical and computer modelling, 18, pp. 85-102.

[5] Cooley, P. C., Hamill, D., Liner, E., Myers, L. and Van Der Horst, C., (1993) A linked risk group model for investigating the spread of HIV, Mathematical and computer modelling, 18, pp. 85-102.

[6] Craig, B. A., Fryback, D. G., Klein, R. and Klein, B. E., (1999) A Bayesian approach to modelling the natural history of a chronic condition from observations with intervention, Statistics in medicine, 18, pp. 1355-1371.

[7] Diekmann, O., Heesterbeek, J. and Metz, J. A., (1990) On the definition and the computation of the basic reproduction ratio $R 0$ in models for infectious diseases in heterogeneous populations, Journal of mathematical biology, 28, pp. 365382.

[8] Dietz, K., (1975) Transmission and control of arbovirus diseases, Epidemiology, pp. 104-121.

[9] Hethcote, H. W., (2000) The mathematics of infectious diseases, SIAM review, 42, pp. 599-653. 
[10] Hethcote, H. W., 1989Three basic epidemiological models, Applied mathematical ecology, Springer, pp. 119-144.

[11] Hiligsmann, M., Ethgen, O., Bruyère, O., Richy, F., Gathon, H. J. and Reginster, J. Y., (2009) Development and validation of a Markov microsimulation model for the economic evaluation of treatments in osteoporosis, Value in health, 12 , pp. 687-696.

[12] Johnson, L., (2004) An Introduction to the mathematics of HIV/AIDS modelling, Capetown: Centre for Actuarial Research, University of Capetown, unpublished manuscript.

[13] Keeling, M. J. and Rohani, P., 2008.Modeling infectious diseases in humans and animals, Princeton University Press, 2008.

[14] Lekone, P. E. and Finkenstädt, B. F., (2006) Statistical inference in a stochastic epidemic SEIR model with control intervention: Ebola as a case study, Biometrics, 62, pp. 11701177.

[15] Mbabazi, D., (2008) Population Dynamic Type Models in HIV Infection, African Institute for Mathematical Science.

[16] Mugisha, J. and Luboobi, L. S., (2003) Modelling the effect of vertical transmission in the dynamics of HIV/AIDS in an agestructured population, The South Pacific Journal of Natural and Applied Sciences, 21, pp. 82-90.

[17] Naresh, R., Tripathi, A. and Omar, S., (2006) Modelling the spread of AIDS epidemic with vertical transmission, Applied Mathematics and Computation, 178, pp. 262-272.

[18] Newell, M.-L., (1998) Mechanisms and timing of mother-to- child transmission of HIV-1, Aids, 12, pp. 831-837.

[19] Newman, M. E., (2002) Spread of epidemic disease on networks, Physical review E, 66, pp. 016128.

[20] Palella Jr, F. J., Delaney, K. M., Moorman, A. C., Loveless, M. O., Fuhrer, J., Satten, G. A., Aschman, D. J. and Holmberg, S. D., (1998) Declining morbidity and mortality among patients with advanced human immunodeficiency virus infection, New England Journal of Medicine, 338, pp. 853-860.

[21] Piot, P. and Bartos, M., 2002The epidemiology of HIV and AIDS, AIDS in Africa, Springer, pp. 200-217.

[22] Schneider, M. F., Gange, S. J., Williams, C. M., Anastos, K., Greenblatt, R. M., Kingsley, L., Detels, R. and Muñoz, A., (2005) Patterns of the hazard of death after AIDS through the evolution of antiretroviral therapy: 1984-2004, Aids, 19, pp. 2009-2018.

[23] Smith, H. L., Wang, L. and Li, M. Y., (2001) Global dynamics of an SEIR epidemic model with vertical transmission, SIAM Journal on Applied Mathematics, 62, pp. 58-69.

[24] Van Den Driessche, P. and Watmough, J., (2002) Reproduction numbers and sub-threshold endemic equilibria for compartmental models of disease transmission, Mathematical biosciences, 180, pp. 29-48.

[25] Venkataramanan, L. and Sigworth, F., (2002) Applying hidden Markov models to the analysis of single ion channel activity, Biophysical journal, 82, pp. 1930-1942.

[26] Who, W. H. O., 2009. 2008 Report on the Global AIDS Epidemic, World Health Organization, 2009. 